\title{
ON THE HOLOMORPH OF A CYCLIC GROUP*
}

BY

\section{G. A. MILLER}

It is known that the holomorph $(K)$ of a cyclic group $(G)$ is a complete group and that its commutator subgroup is $G$ whenever the order $(g)$ of $G$, is odd. When $g>2$ is even, the commutator subgroup of $K$ is the subgroup of $G$ whose order is $g / 2$, and $K$ is never complete. $\dagger$ The main object of this paper is to determine additional useful properties of $K$ whose subgroups are of such fundamental importance. In particular, we shall determine the orders of all the operators of $K$ and some of the properties of its group of isomorphisms when $g$ is even. It will be observed that the generalized Fermat's theorem follows directly from some of the properties of the group of isomorphisms of $G$.

Let $g=2^{\alpha_{0}} p_{1}^{\alpha_{1}} p_{2}^{a_{2}} \cdots p_{m}^{a_{m}}\left(p_{1}, p_{2} ;, \cdots, p_{m}\right.$ being any odd prime numbers) and let $K_{0}, K_{1}, K_{2}, \cdots, K_{m}$ represent the holomorphs of the cyclic groups $\left(G_{0}, G_{1}, G_{2}, \cdots, G_{m}\right)$ of orders $2^{a_{0}}, p_{1}^{a_{1}}, p_{2}^{a_{2}}, \cdots, p_{m}^{a_{m}}$ respectively. As $K$ is evidently the direct product of these holomorphs $\ddagger$ the orders of all the operators of $K$ can be directly obtained from the orders of the operators in these holomorphs. We shall first consider the operators of $K_{0}\left(\alpha_{0}>2\right)$ whose order is known to be $2^{2 \alpha_{0}-1}$.

The group of isomorphisms $\left(I_{0}\right)$ of $G_{0}$ is known to be the direct product of an operator of order two and a cyclic group which may be so chosen that it is composed of all the operators of $I_{0}$ which transform $\S$ an operator of order 4 in $G_{0}$ into itself. 9

The orders of the two independent generators $\left(s_{1}, s_{2}\right)$ of $I_{0}$ are therefore $2^{a_{0}-2}$ and 2 respectively.** We shall first determine the orders of all the

* Presented to the Society (San Francisco) December 20, 1902. Received for publication January 3, 1903.

† Burnside, Theory of groups of finite order, 1897, p. 240 ; Miller, Quarterly Journal of Mathematics, vol. 31 (1899), p. 382.

$\ddagger$ Cf. Burnside, l. c.

§ It will be assumed throughout that $K_{a}(a=0,1,2, \cdots, m)$ is represented as a substitution group whose degree is equal to the order of $G_{a}$, and that its group of isomorphisms $\left(I_{a}\right)$ is the subgroup composed of all the substitutions which omit the first letter. The two groups $K_{a}$ and $I_{a}$ are thus completely determined by $G_{a}$ even as substitution groups.

IB Bulletin of the American Mathematic al Society, vol. 7 (1901), p. 350.

** Since all the squares of operators of $K_{0}$ which occur in $G_{0}$ must also be found in the commutator subgroup of $K_{0}$, it follows that the quotient group of $K_{0}$ with respect to its commutator subgronp is of type $(a-2,1,1)$. 
operators of $K_{0}$ which may be obtained by multiplying $G_{0}$ by powers of $s_{1}$, where $s_{1}$ is supposed to have been so selected that it is commutative with an operator of order 4 in $G_{0}$.

Let $s$ represent any operator of highest order in $G_{0}$. From the equations

$$
\left(s_{1}^{\alpha} s\right)^{2}=s_{1}^{\alpha} s s_{1}^{\alpha} s=s_{1}^{2 a} s_{1}^{-a} s s_{1}^{\alpha} s=s_{1}^{2 a} s^{\prime} s^{2},
$$

where $s^{\prime}$ is some operator of $G_{0}$ whose order is equal to that of $s_{1}^{a}$ and hence $s^{\prime} s^{2}$ is of the same order as $s^{2}$, it follows that $s_{1}^{a} s\left(\alpha=1,2, \ldots, 2^{a_{0}-2}\right)$ is of the same order as $s .^{*}$ Hence $K_{0}$ contains at least $2^{a_{0}-2}$ cyclic subgroups of order $2^{a_{0}}$. We shall soon see that this is the exact number of such subgroups and that, with the exception of $G_{0}$, they are conjugate in sets of

$$
2^{0}, 2,2^{2}, 2^{3}, \ldots, 2^{a_{0}-3} \text {. }
$$

In particular, $K_{0}$ contains just two invariant cyclic subgroups of order $2^{a_{0}}$, so that the holomorph of a cyclic group of order $2^{a_{0}}$ is at the same time the holomorph of just one other cyclic group of this order. All the substitutions which transform one of these subgroups into the other must therefore transform $K_{0}$ into itself and have their squares in $K_{0} \cdot \dagger$

If $s^{2}$ is substituted for $s$ in the equations of the preceding paragraph it follows in a similar manner that $s_{1}^{\alpha} s^{2}$ is of the same order as $s^{2}$ since the order of $s_{1}^{2 a}$ is less than that of $s^{4}$. Hence the group generated by $s_{1}$ and $G_{0}$ contains just $2^{a_{0}-2}$ cyclic subgroups of order $2^{a_{0}-1}$. It will, however, be seen that these are just half of the cyclic groups of this order which are found in $K_{0}$, the other half being obtained by multiplying the group generated by $G_{0}$ and $s_{1}$ by the operator $s_{2}$.

In general, the order of the product of $s_{1}^{a}$ into any operator $s^{\beta}$ of $G_{0}$ is equal to the least common multiple of the orders of the two factors $s_{1}^{a}, s^{\beta}$, for this order cannot be less than $s_{1}^{a}$, since, the product transforms the operators of $G_{0}$ according to a substitution of this order, and from the preceding paragraphs it is clear that it can exceed the order of $s_{1}^{a}$ only when the order of $s^{\beta}$ exceeds that of $s_{1}^{a}$. In this case the order of the product is equal to the order of $s^{\beta}$. Hence the group generated by $G_{0}$ and $s_{1}$ contains $3.2^{2(n-1)}$ operators of order $2^{n}\left(0<n<a_{0}-1\right), 2^{2 a_{0}-4}$ of order $2^{a_{0}-1}$, and $2^{a_{0}-3}$ of order $2^{a_{0}}$.

As each of the remaining operators of $K_{0}$ must transform an operator of order 4 in $G_{0}$ into its inverse and as $G_{0}$ contains negative substitutions, all of these operators must be conjugate in sets of $2^{a_{0}-1}$. If $s_{2}$ has been so selected

* Bulletin, l. c.

+ From this it is clear that $G_{0}$ has always a double holomorph in the same letters as its holomorph. Every non-abelian group has also such a double holomorph since the substitutions which transforms a non-abelian group in to its conjoint must transform this conjoint into the given nonabelian group. Abelian groups do not always bave such double holomorphs. 
that it transforms each operator of $G_{0}$ into its inverse (which will be assumed) all the products obtained by multiplying the operators of $G_{0}$ by $s_{2}$ are of order 2. One half of the products obtained by multiplying $G_{0}$ by $s_{1}^{\beta} s_{2}$ $\left(\beta \neq 0\right.$ mod $\left.2^{a_{0}-2}\right)$ are of the same order as $s_{1}^{\beta}$, since $s_{1}$ and $s_{2}$ are both found in $I_{0}$ and $s_{1}^{\beta} s_{2}$ is of degree $2^{a_{0}}-2$. We proceed to prove that the rest are of twice this order.*

None of the operators in question is commutative with $s_{1}^{2^{a_{0}-3}}$ or its conjugate, for each of these two conjugates is commutative with $s_{1}^{\beta} s_{2}$ and hence with all the products obtained by multiplying $s_{1}^{\beta} s_{2}$ into the positive substitutions of $G_{0}$, but it is commutative with only half the operators of $K_{0}$ since its degree is less than $2^{a_{0}}$. If the order of $s_{1}^{\beta} s_{2}$ is $2^{\gamma}(\gamma>1)$ the $2^{\gamma-1}$ power of the operators in question must therefore be of order 4 ; i. e., their order must be twice the order of $s_{1}^{\beta} s_{2}$. If $\gamma=1, s_{1}^{\beta} s_{2}$ transforms $s$ into its $2^{a_{0}-1}-1$ power and the theorem clearly remains true.

Combining the results of the last two paragraphs it follows that $K_{0}$ contains $3\left(2^{a_{0}-1}+1\right)$ operators of order $2,3\left(2^{2(n-1)}+2^{a_{0}+n-3}\right)$ of order $2^{n}\left(1<n<\alpha_{0}-1\right)$, and $2^{2 a_{0}-3}$ of each of the two orders $2^{a_{0}-1}, 2^{a_{0}}$. This includes the fact that all the operators of order $2^{a_{0}}$ which are contained in $K_{0}$ transform an operator of order 4 in $G_{0}$ into itself as was stated above. Since the products obtained by multiplying all the operators of $G_{0}$ by any given power of $s_{1}$ have the same number of conjugates under $I_{0}$ as the multiplicands have and since any operator of $I_{0}$ has as many conjugates under $G_{0}$ as its order has units, it follows that all the operators of the same order which may be obtained by multiplyiny $G_{0}$ by any one operator of $I_{0}$ with the exception of $s_{2}$ are conjugate under $K_{0} . \dagger$ In particular, the cyclic subgroups of order $2^{a_{0}}$ are conjugate in sets of $2^{0}, 2^{0}, 2,2^{2}, \ldots, 2^{a_{0}-3}$ as was stated above.

In what precedes it has been explicitly assumed that $\alpha_{0}>2$. When $\alpha_{0}=2$, $K_{0}$ is the well known octic group and includes only one cyclic subgroup of order $2^{\alpha_{0}}$. All its other operators are of order 2. When $\alpha_{0}=1, K_{0}$ coincides with $G_{0}$. It remains to determine the orders of all the operators of $K_{1}$. It will be seen that the order of the product of any operator of $G_{1}$ into any operator whose order is of the form $p_{1}^{\beta}$ in $I_{1}$ is the least common multiple of the orders of the two factors. Hence this case is similar to the first part of the preceding case. There is, however, only one invariant cyclic subgroup of order $p_{1}^{a_{1}}$ in $K_{1}$, as the $p_{1}-1$ subgroups of this order which correspond to the second invariant subgroup in $K_{0}$ are conjugate under $K_{1}$. We proceed to prove these statements.

* Their orders could not exceed this number since they are rot commutative with the operators of order 4 in $G_{0}$.

† That these operators form complete sets of conjugates under $K_{0}$ follows directly from the fact that $K_{0} / G_{0}$ is abelian. The products of $G_{0}$ and $s_{2}$ clearly form two complete sets of conjugates under $K_{0}$. 
Let $s$ represent any operator of $G_{1}$ and let $t$ represent any operator whose order is of the form $p_{1}^{\beta}$ in $I_{1}$. Suppose that

$$
t s t^{-1}=s_{1} s, t s_{1} t^{-1}=s_{2} s_{1}, \cdots, t s_{a} t^{-1}=s_{a+1} s_{\alpha} .
$$

It is known that the order of $s_{a+1}$ is lower than that of $s_{a} \cdot{ }^{*}$ From the equations

$$
\begin{aligned}
(s t)^{n} & =s t s t^{-1} t^{2} s t^{-2} t^{3} s \cdots t^{n-1} s t^{1-n} t^{n} \\
& =s s_{1} s s_{2} s_{1}^{2} s \cdots s_{n-1} s_{n-2}^{n-1} \cdots s_{n-r-1}^{\frac{(n-1) \ldots(n-r)}{r !}} \cdots s_{1}^{n-1} s t^{n} \\
& =\cdots s_{1}^{2-\frac{n-1)}{2}} s^{n} t^{n}
\end{aligned}
$$

where the omitted factors in the last member are of a lower order than $s_{1}$, it follows that $s t$ is of the same order as it would be if $s$ and $t$ were commutative and independent. That is, the order of $s t$ is the least common multiple of the orders of $s$ and $t$. If the order of $t$ is not of the form $p_{1}^{\beta}$ then $s t$ is of the same order as $t$ since $t$ is not commutative with any operator of $G_{1}$ besides the identity and $I_{1}$ is abelian.

From the results of the preceding paragraph we can readily determine the number of operators of a given order in $K_{1}$. 1here are just $\phi(n) p_{1}^{a_{1}}$ operators of order $n$ in $K_{1}, n$ representing any divisor of $p_{1}^{a_{1}-1}\left(p_{1}-1\right)$ which is not of the form $p_{1}^{\beta}$. The orders of the remaining operators are of the form $p_{1}^{\beta}$ and there are $p_{1}^{2(\beta-1)}\left(p_{1}^{2}-1\right)$ of this order whenever $\beta<\alpha_{1}$. When $\beta=\alpha_{1}$ there are $p_{1}^{2\left(a_{1}-1\right)}\left(p_{1}-1\right)$ operators of order $p^{\beta}$. In exactly the same manner as in the preceding case it may be seen that all the operators of the same order which may be obtained by multiplying the operators of $\left[G_{1}\right.$ by any one operator of $I_{1}$ form a complete set of conjugates under $K_{1}$. Hence the cyclic subgroups of order $p_{1}^{a_{1}}$ in $K_{1}$ are conjugate in sets of

$$
1, p_{1}-1, p_{1}\left(p_{1}-1\right), \cdots, p_{1}^{a_{1}-2}\left(p_{1}-1\right) \text {. }
$$

These results can readily be applied to the holomorph $K$ of the general cyclic group $G$. In the first place, $K$ contains only one invariant cyclic subgroup of order $g$ whenever $g$ is not divisible by 8 . If $g$ is divisible by 8 then $K$ contains just two such subgroups, having $g / 2$ common operators. This result follows directly from the facts that $K_{1}$ contains only one invariant cyclic subgroup of order $p_{1}^{a_{1}}$ and that $K_{0}$ contains one or two invariant cyclic groups of order $2^{\alpha_{0}}$ according as $\alpha_{0}<3$ or $\alpha_{0} \equiv 3$. The group of isomorphisms of $G$ will be denoted by $I$ and it will be assumed that $K$ is represented as a transitive substitution group of degree $g$. Hence $G$ must be regular. $\dagger$ Let $t$ represent the

* Bulletin of the American Mathematical Society, vol. 7 (1901), p. 351.

† If a transitive group of degree $n$ contains an invariant cyclic subgroup of order $n$ this cyclic subgroup must be regular. If the subgroup were non-cyclic it would not need to be regular. 
substitution of $I$ which transforms a generator $s$ of $G$ into its $\alpha^{\prime}$ th power. The orders of all the substitutions of $K$ which transform $s$ into its $\alpha$ power are the same as those of the direct product of the divisions in the holomorphs $K_{0}, K_{1}, \ldots, K_{m}$ which transform the generators of $G_{0}, G_{1}, \ldots, G_{m}$ respectively in the same manner as $t$ does. The number of sets of conjugates among these $g$ substitutions is clearly equal to the product of the numbers of the sets of conjugates in the given divisions of $K_{0}, K_{1}, \cdots, K_{m}$.

The following examples may serve to exhibit more clearly some of the properties mentioned above. If $g=100$ and $\alpha=9$ the orders of the operators obtained by multiplying $G$ into $t$ are the same as those in the direct product of the cyclic group of order 4 and the division in the holomorph of the cyclic group of order 25 which transforms the operators of this cyclic group into their 9 th powers. As all the operators of the latter division are of order 10, $t$ is of order 10 and has 25 conjugates under $K$. There is another set of 25 conjugates of this order, while the remaining 50 operators of $K$ which transform $s$ into the 9th power are of order 20 and from a single set of conjugates under $K$. If $g=100$ and $\alpha=3$ there are evidently two equal sets of conjugates, each of the 100 operators being of order 20 .

It is very easy to determine the degrees of all the substitutions of $K$. Since the substitution $t$ is in $I$ its degree is less than $g$. We may suppose that it omits the first letter of $G$. If it omits any other letter it must be commutative with the substitution in which the first letter is replaced by this second. That is, if $t$ is of degree $g-\beta$ it is commutative with just $\beta$ substitutions of $G$. The number of its conjugates under $G$ (which is clearly equal to the number of its conjugates under $K$ ) is $g / \beta$. All the other products obtained by. multiplying $G$ by $t$ must be of degree $g$, otherwise $K$ would contain a transitive subgroup in which the average number of letters would not be $g-1$. $^{*}$ It is clear that the condition in regard to the average number of letters in the substitutions of a transitive group requires that in each division of $K$ with respect to $G$ this average number is $g-1$.

The result of the preceding paragraph may be stated as follows : If any subtitution $t$ of $I$ is commutative with just $\beta$ substitutions of $G$ its degree is $g-\beta$ and it has $g / \beta$ conjugates under $K$. All the products obtained by multiplying $G$ by $t$ are of degree $g$, with the exception of these $g / \beta$ conjugates of $t$. For instance, in the first example of the next to the preceding paragraph, there are 25 substitutions of degree 96 and 75 of degree 100 . In the second example there are 50 of degree 98 and 50 of degree 100.

It is evident that $I$ can be represented as a regular group of degree $\phi(g)$ since each of its substitutions must permute all the generators of $G$ and these generators can be permuted transitively. In fact, this regular group is one of

* Cf. Bulletin of the American Mathematical Society, vol. 2 (1895), p. 75. 
the transitive constituents of $I$ and each of its other constituents is also regular. As $I$ is the group formed by combining the $\phi(g)$ numbers by multiplication and replacing the products by their least positive residues modulo $g$, Fermat's generalized theorem $\left(a^{\phi(g)} \equiv 1 \bmod g\right)$ is merely a statement of the fact that the order of $I$ is divisible by the order of each of its substitutions.

When $g=p^{a}, p$ being any odd prime, $I$ contains $\alpha$ transitive constituents whose orders are $p^{\beta}(p-1), \beta=0,1, \ldots, \alpha-1$, respectively. If $\gamma>\delta$ then the constituent of order $p^{\gamma}(p-1)$ has a $\left(p^{\gamma-\delta}, 1\right)$ isomorphism with the constituent of order $p^{\delta}(p-1) \cdot \dagger$ Hence $I$ contains just $p-1$ substitutions of degree $\phi(g)$ and its structure as a substitution group is completely determined. When $p=2 I$ contains only $\alpha-1$ transitive constituents and the given subgroup of order $p^{\gamma-\delta}$ is generated by a substitution corresponding to $s_{1}$ in $I_{0}$ as used above. Hence in this case $I$ is also completely determined as a substitution group.

It has been observed that $I$ always contains one transitive constituent of order $\phi(g)$. When $\alpha_{0}=1$ there is one more constituent of this order. In all other cases there is only one such constituent since the group of isomorphisms of every subgroup of $I$ is of a lower order than $I$. When $\alpha_{0}=0$ there are $\left(\alpha_{1}+1\right)\left(\alpha_{2}+1\right) \cdots\left(\alpha_{m}+1\right)-1$ transitive constituents in $I$ while there are $\left(\alpha_{0}+1\right)\left(\alpha_{1}+1\right) \cdots\left(\alpha_{m}+1\right)-2$ such constituents in all other cases. $\ddagger$ As $\phi(g)$ is the order of the constituent formed by the direct product of the constituents representing the permutations of the operators in the subgroups of orders $2^{a_{0}}, p_{1}^{a_{1}}, \cdots, p_{m}^{a_{m}}$ respectively it follows that $I$ contains substitutions of degree $\phi(g)$ only when $g=p^{a}$.

The preceding results are sufficient to determine $I$ as a substitution group. It is only necessary to determine the isomorphisms between the given regular constituent of order $\phi(g)$ and the remaining regular constituents. As each of these constituents represents the permutation of the operators of highest order in some subgroup of $G$, the required isomorphism is equivalent to determining all the permutations of the operators of highest order in $G$ which do not affect the generators of this subgroup. The latter can be directly obtained from the powers of the primes which enter into the order of the subgroup.

It has been observed that the group of isomorphisms $\left(I^{\prime}\right)$ of $K$ is the same as $K$ itself whenever the order $(g)$ of $G$ is odd. We proceed to find the order of $I^{\prime}$ when $g$ is even. Representing $K$ as an intransitive substitution group whose transitive constituents are $K_{0}, K_{1}, \cdots, K_{m}$, we shall first determine what groups may correspond in the holomorphisms of $K$ to $K_{a}$, $\alpha$ having any one of

* Annals of Mathematics, vol. 2 (1900), p. 77.

† Bulletin of the American Mathematical Society, vol. 7 (1901), p. 350.

$\ddagger$ Cf. Dirichlet, Zahlentheorie, 1894, p. 17. 
the values $1,2, \ldots, m$. Since every subgroup of $G^{*}$ is a characteristic subgroup of $K$ the group which corresponds to $K_{a}$ must involve all the letters of $K_{a}$. As $K_{a}$ is a complete group the corresponding group must be either $K_{\alpha}$ itself or it must involve $K_{a}$ as one of its transitive constituents. We proceed to prove that in the latter case the other constituents must be the invariant substitution $\left(S^{\prime}\right)$ of $K$, which is not the identity.

This follows immediately from the fact that each of the substitutions of $K_{\alpha}$ is commutative with all the substitutions in the direct product of the remaining partial holomorphs $K_{0}, K_{1}, \cdots, K_{m}$. As the corresponding group must have the same property with respect to a similar group it is proved that in any simple isomorphism of $K$ with itself each of the partial holomorphs $K_{1}, K_{2}, \ldots, K_{m}$ either corresponds to itself or to the group obtained by multiplying half its operators by $S^{\prime}$. In these holomorphisms $S^{\prime}$ cannot be multiplied into an operator of $G$ and hence $K_{\alpha}$ can correspond to two and only two subgroups of $K$. The partial holomorph $K_{0}$ must always correspond to itself.

From the preceding paragraph it follows that $I^{\prime}$ contains a subgroup of order $2^{m}$ which includes no operator whose order exceeds 2 and which has only the identity in common with $I^{\prime \prime}$ the group of cogredient isomorphisms of $K$. Each of these $2^{m}$ operators is commutative with every operator of $I^{\prime \prime}$ according to the following evident theorem: Any operator of the group of isomorphisms which corresponds to a holomorphism obtained by multiplying half the operators of the group by an invariant operator of order 2 is commutative with every operator in the group of cogredient isomorphisms. Hence $I^{\prime}$ must always include the direct product of $I^{\prime \prime}$ and this abelian group of order $2^{m}$. When $\alpha_{0}=1$ it is clear that $I^{\prime}$ contains no operators besides this direct product, and when $\alpha_{0}=2$ the order of $I^{\prime}$ is twice the order of this direct product. We proceed to prove that for all other values of $\alpha_{0}$ the order of $I^{\prime}$ is four times the order of this direct product.

To prove this it is only necessary to determine the order of the group $\left(I_{0}^{\prime}\right)$ of isomorphisms of $K_{0}$. Consider the divisions of $K_{0}$ with respect to $G_{0}$. One of these contains $2^{a_{0}-1}$ substitutions of order $2^{a_{0}-2}$ and of degree $2^{a_{0}}-2$ while its remaining substitutions are of degree $2^{\alpha_{0}}$ and of order $2^{a_{0}-1}$. The substitutions of each of these two sets must correspond to themselves whenever $G_{0}$ corresponds to itself. As any two substitutions, one from each of these sets, generate one half of $K_{0}$ and as the division which transforms each operator of $G_{0}$ into its inverse contains only two substitutions which are commutative with a substitution of the first set, it follows that the number of the operators in $I_{0}^{\prime}$, which transform $G_{0}$ into itself, cannot exceed $2^{a_{0}-1} \cdot 2^{a_{0}-1} \cdot 2$. Hence the order of $I_{0}^{\prime}$ cannot exceed $4 \cdot 2^{2\left(a_{0}-1\right)}$; i. e. four times the order of the group $\left(I_{0}^{\prime \prime}\right)$ of cogredient isomorphisms of $K_{0}$.

* When $g$ is divisible by $8, G$ itself is not a characteristic subgroup of $K$. In this case $G$ has a double holomorph of degree $g$. 
It remains only to prove that $I_{0}^{\prime}$ contains operators which transform $G_{0}$ into itself but are not found in $I_{0}^{\prime \prime}$. Such an operator of order 2 corresponds to the simple isomorphism of $K_{0}$ which may be obtained by multiplying by $S^{\prime}$ all the operators obtained by multiplying $G_{0}$ by all the operators of order $2^{a_{0}-2}$ in $I_{0}$ in order. Hence the theorem: The order of the group of isomorphisms of $K$ is $2^{m}, 2^{m+1}$ or $2^{m+2}$ times the order of its group of cogredient isomorphisms as $\alpha_{0}=1,2$, or $>2 . *$

* Cf. Burnside, Theory of groups of finite order, 1897, p. 242. 